The Astrophysical Journal, 683:566-576, 2008 August 10

(C) 2008. The American Astronomical Society. All rights reserved. Printed in U.S.A.

\title{
MULTISCALE ASTRONOMICAL IMAGE PROCESSING BASED ON NONLINEAR PARTIAL DIFFERENTIAL EQUATIONS
}

\author{
Meyer Pesenson, William Roby, and Bruce McCollum \\ Spitzer Science Center, California Institute of Technology, MC 314-6, Pasadena, CA 91125; misha@ipac.caltech.edu \\ Received 2007 December 4; accepted 2008 April 11
}

\begin{abstract}
Astronomical applications of recent advances in the field of nonastronomical image processing are presented. These innovative methods, applied to multiscale astronomical images, increase signal-to-noise ratio, do not smear point sources or extended diffuse structures, and are thus a highly useful preliminary step for detection of different features including point sources, smoothing of clumpy data, and removal of contaminants from background maps. We show how the new methods, combined with other algorithms of image processing, unveil fine diffuse structures while at the same time enhance detection of localized objects, thus facilitating interactive morphology studies and paving the way for the automated recognition and classification of different features. We have also developed a new application framework for astronomical image processing that implements some recent advances made in computer vision and modern image processing, along with original algorithms based on nonlinear partial differential equations. The framework enables the user to easily set up and customize an image-processing pipeline interactively; it has various common and new visualization features and provides access to many astronomy data archives. Altogether, the results presented here demonstrate the first implementation of a novel synergistic approach based on integration of image processing, image visualization, and image quality assessment.
\end{abstract}

Subject headings: astrometry — diffusion — methods: data analysis — methods: numerical — techniques: image processing

\section{INTRODUCTION}

During the last 20 years the image processing community outside of astronomy has made substantial progress in developing powerful methods for computer vision and image processing (Mitra \& Sicuranza 2001; Forsyth \& Ponce 2003; Bovik 2005; Paragios et al. 2006), which for the most part have not yet been utilized by the astronomy community. Multiscale image representation and enhancement have become important parts of computer vision systems and modern image processing and have recently begun to be applied in astronomy (Starck et al. 1998, 2002). This approach has proven to be especially useful for feature detection. Supporting interactive or semiautomated processing, especially in a time when many astronomical data sets are increasing greatly in size, demands a new approach based on employing innovative methods of modern image processing and integrating them with visualization and image quality assessment. This paper describes such a novel synergistic approach.

Adapting recent advances of computer vision and image processing for astronomy, and especially designing and implementing an advanced image processing framework that would utilize these continuing achievements, remains, however, a major challenge. The keystone elements of such a system that unifies a wide range of methods should be computational and visualization modules. Developers and users alike have realized that there is more to creating an application than simple programming. The objective of creating a flexible (see below) application is customarily achieved by exploiting the object-oriented paradigm (Grand 1998; Booch et al. 2005). In computer science such systems are called frameworks (Fayad et al. 1999). Users can extend frameworks' capabilities by installing plug-ins. An application framework consists of computational (processing) modules, data, and an interactive interface. Frameworks are extendable (i.e., they can easily adopt software products to changes of specification) and reusable (the software elements are able to serve for construction of many different new applications).
The structure of the paper is as follows. In $\oint 2$ we discuss image enhancement and multiscale representation of images based on nonlinear partial differential equations (PDEs) and their application to object detection. Image processing operators that facilitate morphology studies when combined with the multiscale processing from $\S 2$ are discussed in $\S 3$. Section 4 is dedicated to detection of artifacts and image quality assessment, followed by a brief description of the framework. The paper ends with conclusions and plans for future work.

\section{IMAGE ENHANCEMENT AND MULTISCALE REPRESENTATION OF IMAGES WITH NONLINEAR PARTIAL DIFFERENTIAL EQUATIONS}

Image enhancement is an area of image processing that consists of a task-dependent collection of methods that accentuate the features important for a specific objective, thus making human or automated analysis more effective (e.g., Gonzalez \& Woods 2002). It is hard to list all possible applications of image enhancement, so let us mention just a couple of cases where enhancement is required. Astronomical images often contain many point sources and, at the same time, extended diffuse structures with imbedded point sources; all these features are usually degraded by noise, so noise reduction is one of the steps necessary for detection of different objects. In studies of morphology of the galaxy distribution, smoothing is required to construct a real density field (Martínez \& Saar 2002; Martínez et al. 2005). These two examples demonstrate that image enhancement is an important precursor to extraction of useful information from images. In this section we focus on multiscale representation and denoising of images by using PDEs.

Applying traditional smoothing methods such as convolution with a Gaussian inevitably erases small-scale objects. Moreover, for morphological studies of galaxy distribution, smoothing on scales larger than the scale at which the galaxy clustering correlation length is significant produces a Gaussian distribution by 

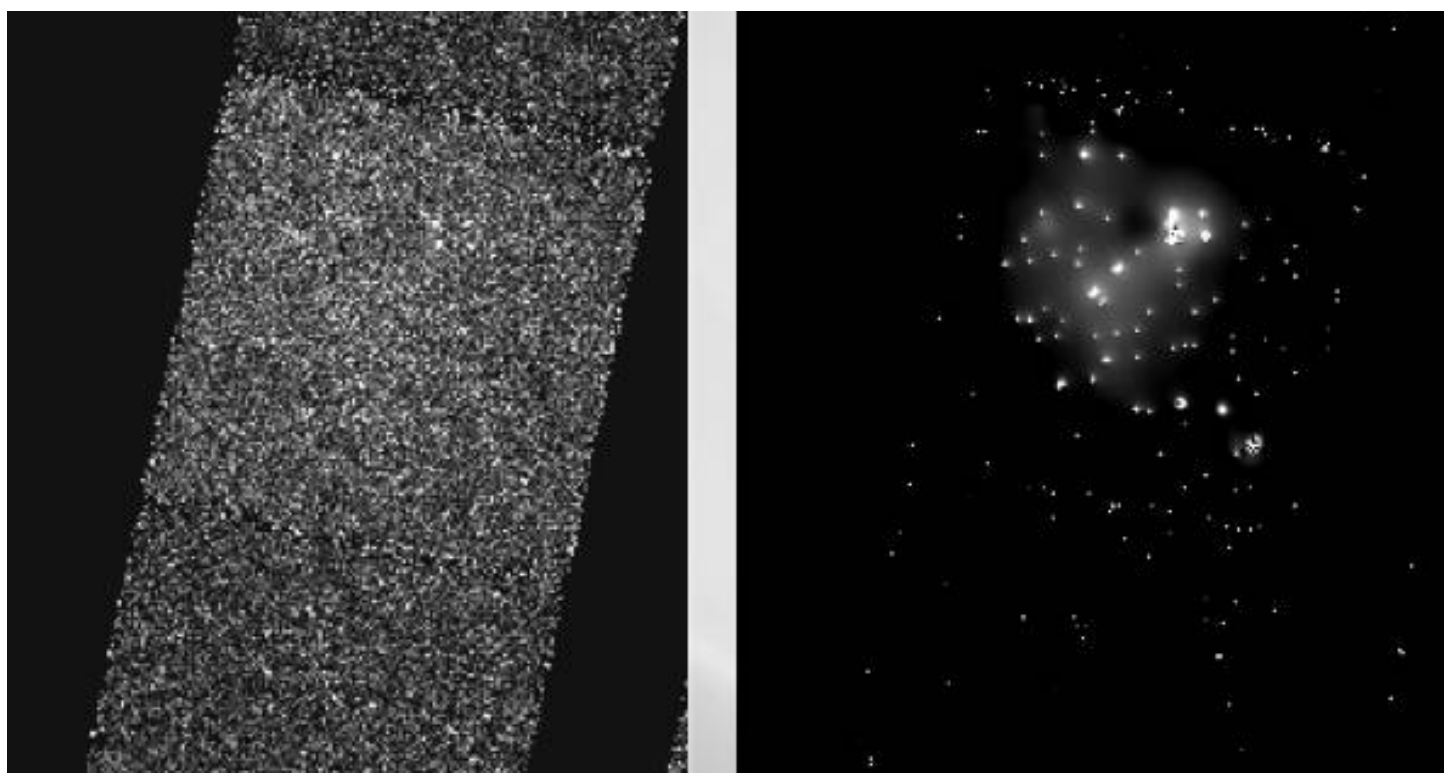

FIG. 1.-Left: Supernova remnant W28 image, Chandra X-Ray Observatory (courtesy of J. Rho [SSC, Caltech]). Right: W28 image processed by the NLD. The brightness is proportional to the flux (logarithmic scale).

virtue of the central limit theorem (Coles \& Lucchin 1995; Martínez et al. 2005; Martínez 2008). Thus, even though convolution with a Gaussian is effective at removing noise, it has the unwanted side effect of eliminating tiny objects, smearing more prominent ones, blurring boundaries of extended structures, etc.

There are two main approaches to the problem of smearing: wavelets (Starck et al. 1998, 2002; Martínez \& Saar 2002; Martínez et al. 2005; Martínez 2008; Ingalls et al. 2004) and methods based on PDEs (Sapiro 2001 and references therein; Lenzen et al. 2004; Acton 2005 and references therein; Pesenson et al. 2005a, 2005b, 2007, 2008). In fact, these two approaches are complementary to each other, and there are a number of connections between them (Paragios et al. 2006). The framework presented in this paper is based on nonlinear PDEs and, in particular, on a nonlinear diffusion (NLD) equation, which is discussed in this section. It should be mentioned that the tradeoff between smoothing and preserving objects is inevitable, and a balance between these two desirable, but conflicting objectives depends on the specific task.
The convolution of an image with a Gaussian is equivalent to solving a Cauchy problem (an initial value problem) for the linear PDE of diffusion with the noisy image as an initial condition (Witkin 1983); this explains the blurring of boundaries, as one would expect from diffusion. This insight has led to the construction of multiscale representations of image data.

Multiscale representation (Witkin 1983; see also Starck et al. $1998,2002)$ is defined as embedding a given image into a oneparameter family of derived images that begins with the original, fine-scale image (see the initial condition in eq. [1]) and progresses toward more coarse scales. Linear multiscale representation can be achieved by employing the aforementioned connection between image processing and linear equation of diffusion. Starting with the pioneering works of Rudin (1987) and Perona \& Malik (1987), filtering based on nonlinear multiscale representation with nonlinear PDEs has become widely used in nonastronomical image enhancement and object detection. This state-of-the-art approach is based on the design and analysis of nonlinear PDEs. Perona \& Malik proposed a nonlinear diffusion equation with the
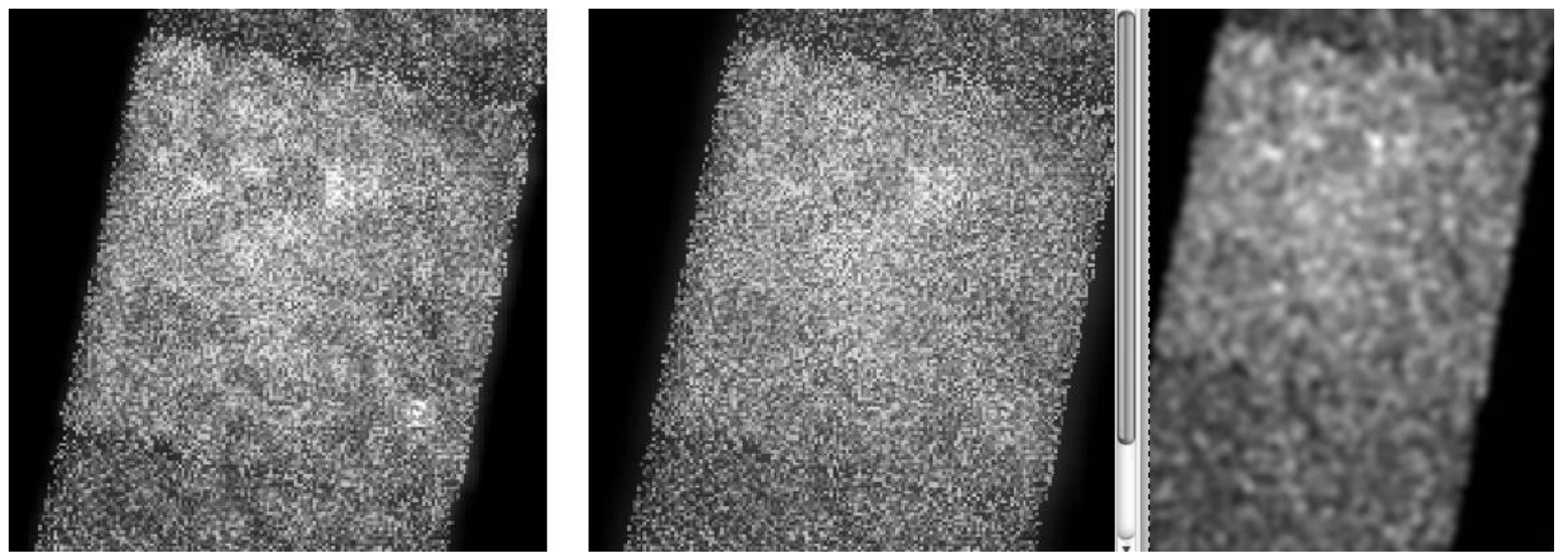

FIG. 2. - Left: W28 image, low-pass average filter, window size $=3 \times 3$. Middle: Same, but with window size $=19 \times 19$. Right: W28 image convolved with a Gaussian ( kernel size $=7$ ). Neither of the approaches provides a noticeable improvement. The brightness is proportional to the flux (logarithmic scale). 


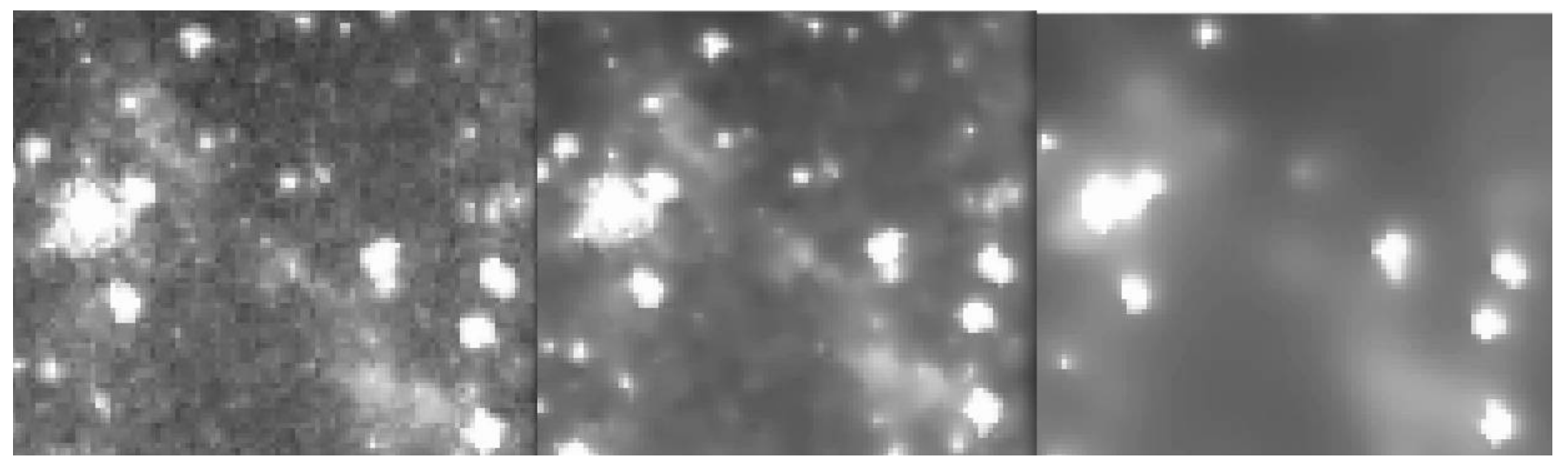

FIG. 3.-Left: G11.2-0.3, Spitzer IRAC $8.0 \mu \mathrm{m}$, GLIMPSE, R. Benjamin (courtesy of W. Reach [SSC, Caltech]). Middle: G11.2-0.3, median filter (window size = 5); the original image is smoothed, but many tiny point sources are lost. Right: G11.2-0.3 image processed by the NLD; signal-to-noise ratio has been increased and point sources, including the ones embedded in a supernova remnant, have been preserved. The brightness is proportional to the flux (logarithmic scale).

coefficient of diffusion $D$ decreasing when the gradient grows and increasing when the gradient decays

$$
\begin{array}{r}
u_{t}=\operatorname{div}\left[D\left(\|\nabla u\|^{2}\right) \nabla u\right], \\
u(0, x, y)=u_{0}(x, y),
\end{array}
$$

where $u(t, x, y)$ is the evolved image at the time $t$, and $u_{0}(x, y)$ is the image to be processed. The gradient $\nabla u$ provides information about local intensity variations (for more information see $\S 3$ ). The coefficient of diffusion $D$ is defined by the following expressions:

$$
D\left(\|\nabla u\|^{2}\right)=\exp \left(-\|\nabla u\|^{2} k^{-2}\right)
$$

or

$$
D\left(\|\nabla u\|^{2}\right)=1 /\left(1+\|\nabla u\|^{2} k^{-2}\right) .
$$

Parameter $k$ characterizes the gradient scale of the initial image. There is no "optimal" value for $k$, and its magnitude depends on the problem. There are many different ways of defining the scale. For the images in this paper we used a quintile in the cumulative gradient histogram (Perona \& Malik 1987).

The aforementioned family of images is parameterized by a scale parameter $t$ (time variable in eq. [1]) and is generated in such a way that fine-scale structures are successively suppressed when the scale parameter is increased. Thus, a multiscale representation allows one to obtain a separation of the image structures in the original image, such that fine-scale image structures only exist at the finest scales in the multiscale representation, thus simplifying the task of object detection. It should be mentioned that the coefficients of diffusion defined above also result in sharpening edges due to the "backward" diffusion (Perona \& Malik 1987), thus also facilitating detection of objects.

Since the work of Perona \& Malik, many substantial modifications of the model have been proposed. Catte at al. (1992) suggested a modification where the diffusion coefficient is evaluated on a presmoothed version of the original image, which makes the method more robust against noise in the data. Since the nonlinear diffusion stops at the edges, noise in these regions remains. To correct this situation, Weickert (1998) proposed an approach where the diffusion is applied parallel to edges.

A different approach to denoising was suggested by Rudin et al. (1992). They proposed a constrained optimization type of numerical algorithm in which the total variation of the image was minimized subject to constraints involving the statistics of the noise.

In order to optimally utilize the "forward/backward" diffusion, one needs to decide when to stop smoothing. This decision becomes very subjective without a quantitative criterion. Indeed, the original image is repeatedly smoothed as the number of iterations increases (i.e., the variable $t$ in eq. [1] increases). To resolve this problem, the reaction term $\beta\left(u-u_{0}\right)$ was introduced

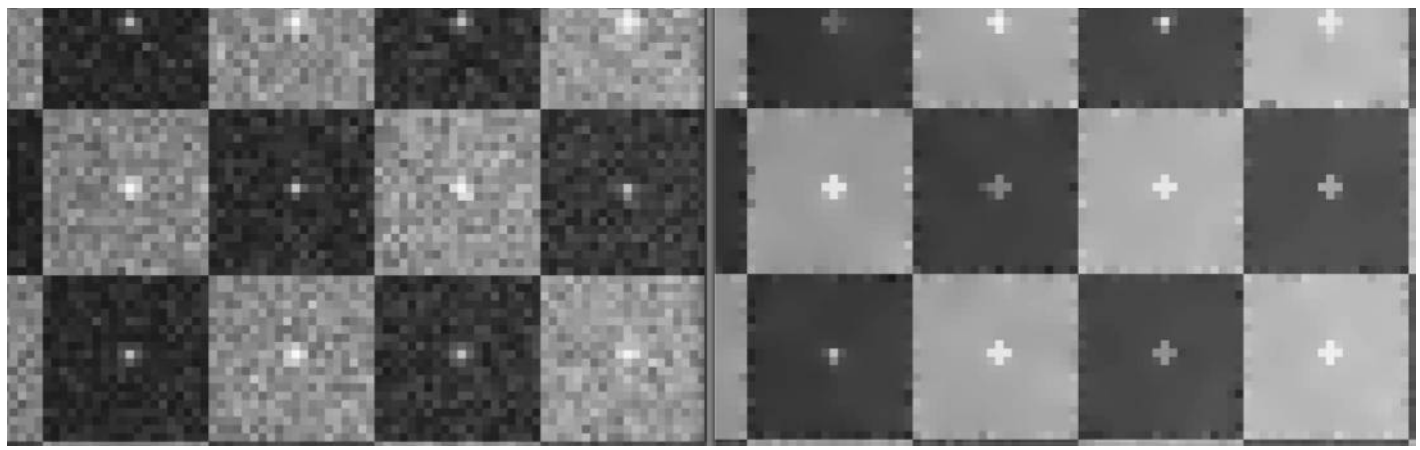

FIG. 4.- Left: Simulated noisy image of a chessboard with localized objects at the center of each square. Right: Simulated noisy image of a chessboard processed by the NLD. Nonlinear diffusion stops exactly at the boundaries; noise level has been reduced while the boundaries remain sharp and localized objects recovered. The brightness is proportional to the flux (logarithmic scale). 


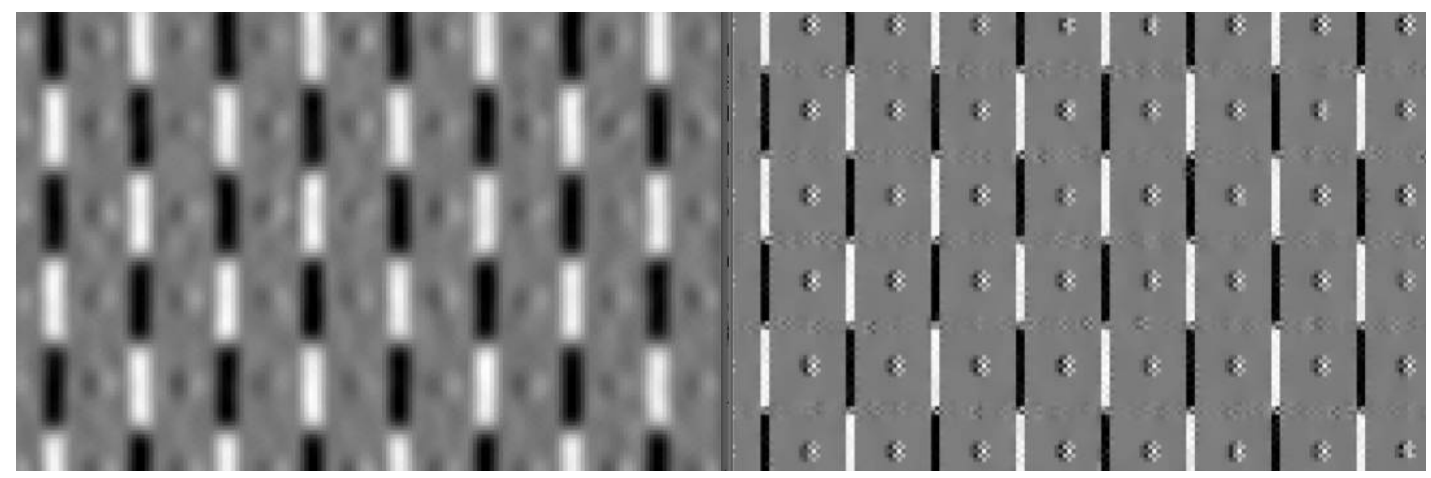

FIG. 5.-Detecting vertical boundaries and point sources. Left: After convolving the noisy image (Fig. 4, left) with a Gaussian followed by detecting vertical lines and point sources, lines are blurred and point sources are drastically smeared. Right: After processing the noisy image (Fig. 4, left) with the NLD module followed by detecting vertical lines, both the linear boundaries and the point sources are recovered much better than after Gaussian preprocessing.

into the nonlinear diffusion equation, with the parameter $\beta$ characterizing the noise level (Rudin et al. 1992; Pesenson et al. 2005a). The reaction term prevents deviation of the smoothed image from the original one by more than the estimated noise. Pesenson et al. (2005a) also used a variable characteristic scale $k$.

A recent effective formulation of nonlinear diffusion for multicomponent images by Tschumperle \& Deriche (2005) opened the possibility of applying this equation to denoising of multiand hyperspectral images. Chao \& Tsai (2006) dealt with what they called "nebulae obscured by stars"; their modification incorporated the variance of gray levels into the diffusion coefficient, thus allowing one to filter out the stars while preserving the nebula. Our goal here is different, and we are mostly concerned with preserving both point sources and diffuse structures.

An example of filtering based on the nonlinear diffusion equation (1) with the exponential coefficient of diffusion is given in Figure 1. Figures 1, 2, and 3 clearly demonstrate that the usual low-pass average filtering or convolution with a Gaussian are not nearly as effective as the NLD. Figure 4 (left) displays a simulated noisy image of a chessboard. Figure 4 (right) demonstrates how nonlinear diffusion, when applied to the simulated image (Fig. 4, left), stops exactly at the boundaries, while the inner areas are all "cleaned up" and the localized objects at the centers of the squares have all been recovered. Thus, preprocessing the simulated image (Fig. 4, left) by applying the NLD enabled a reliable detection of both the vertical boundaries and localized objects (cf. Fig. 5, left and right panels).
Denoising based on nonlinear PDEs improves the quality of point source detection, as was demonstrated by using the completeness and reliability test (Pesenson et al. 2005a). Filtering based on the NLD equation (1) is also employed in our framework for estimating the amount of noise in images (see $\S 4$ ).

Once again, the principal objective of different filters is to prepare an image for the next level of processing, depending on a specific application. Detection of point sources and analysis of morphology of diffuse structures lead to the second level of processing.

\section{IMAGE ENHANCEMENT AND MORPHOLOGY UNVEILING}

The second-level operators implemented in our framework include shape detectors, noise estimation, edge detectors, etc. (Gonzalez \& Woods 2002; Bovik 2005). In this section we discuss some of them, for example, Sobel, Prewitt, and Laplacian of Gaussian (LOG), which are used in image processing for detecting edges of objects in images. Artifacts in astronomical images often have sharp edges, so these operators, combined with shape detectors, facilitate detection of such artifacts (§ 4).

The gradient vector gives the directional difference in intensity between pixels, and it is defined by the following expression:

$$
\nabla u=\left(u_{x}, u_{y}\right)
$$
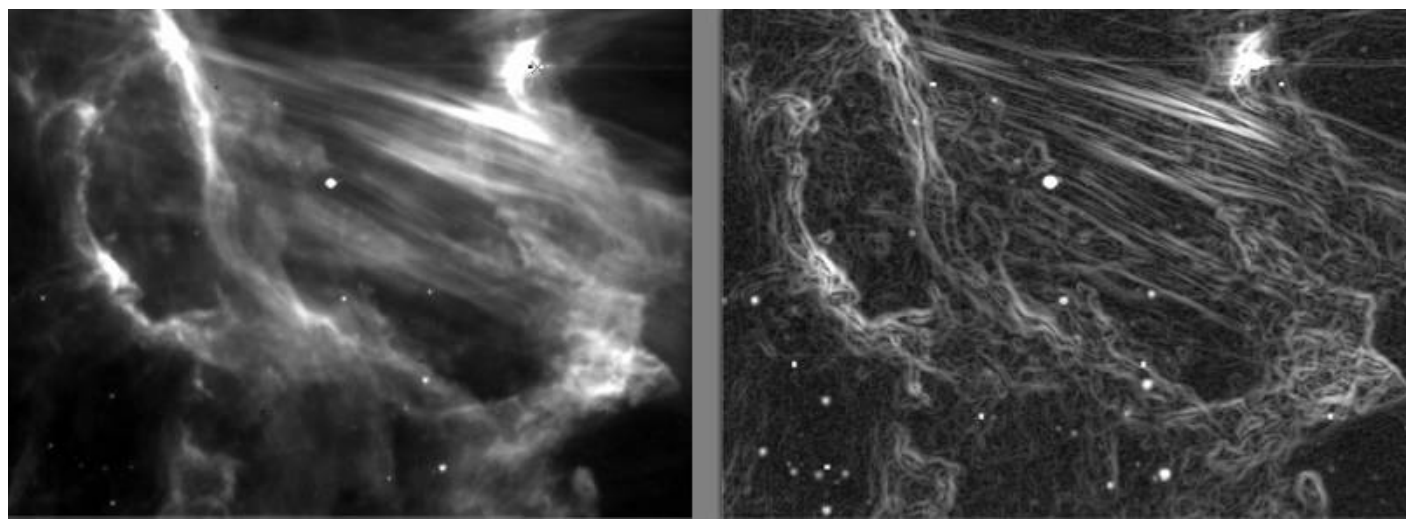

FIG. 6.- Morphology unveiling. Left: IC 405, Spitzer IRAC $8.0 \mu$ m image (France et al. 2007); filaments and a bow shock near HD 34078 (red cross). The brightness is proportional to the flux (logarithmic scale). Right: Brightness is proportional to the module of the gradient of flux (logarithmic scale); this way of looking at astronomical images facilitates analysis of nebular morphology, outflows, jets, embedded sources, and shock fronts. 


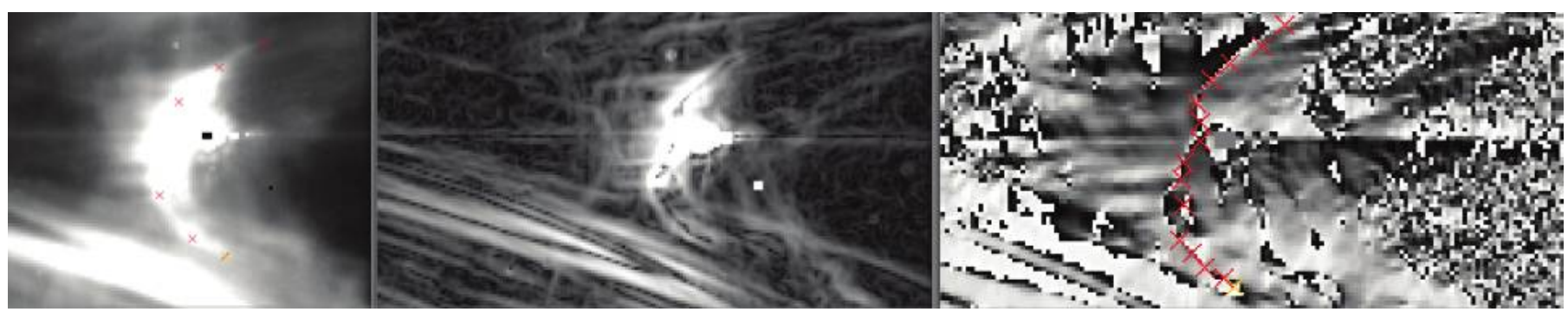

Fig. 7.- Three different visualizations of the bow shock structure. Left: IC 405, Spitzer IRAC $8.0 \mu \mathrm{m}$ image, the bow shock (red crosses) near HD 34078. The brightness is proportional to the flux (logarithmic scale). Middle: The bow shock area where the brightness is proportional to the module of the gradient offlux (logarithmic scale). Right: The bow shock area where the front of the bow shock is immediately apparent as a curve of 1 pixel width (red crosses); the brightness is proportional to the angle of the gradient of flux (logarithmic scale).

Its module is

$$
\|\nabla u\|=\sqrt{u_{x}^{2}+u_{y}^{2}}
$$

Its local direction is defined by the following:

$$
\theta=\arctan \left(\frac{u_{y}}{u_{x}}\right)
$$

The Sobel and Prewitt methods of edge detection are basically different approaches to estimation of the gradient with the aid of convolution masks (Gonzalez \& Woods 2002).

The Sobel masks to be convolved with the image have the following form

$$
u_{x}=\left(\begin{array}{lll}
-1 & 0 & 1 \\
-2 & 0 & 2 \\
-1 & 0 & 1
\end{array}\right), \quad u_{y}=\left(\begin{array}{ccc}
1 & 2 & 1 \\
0 & 0 & 0 \\
-1 & -2 & -1
\end{array}\right)
$$

The Prewitt masks have the following form:

$$
u_{x}=\left(\begin{array}{ccc}
-1 & 0 & 1 \\
-1 & 0 & 1 \\
-1 & 0 & 1
\end{array}\right), \quad u_{y}=\left(\begin{array}{ccc}
1 & 1 & 1 \\
0 & 0 & 0 \\
-1 & -1 & -1
\end{array}\right)
$$

The Prewitt masks give the weights for the best-fitting plane approximating the intensity in a $3 \times 3$ neighborhood, assuming all nine samples have equal weight.

The discrete implementation of the Laplacian has the following form:

$$
\frac{1}{4}\left(\begin{array}{ccc}
0 & -1 & 0 \\
-1 & 4 & -1 \\
0 & -1 & 0
\end{array}\right)
$$

Using these operators can bring out complex structures that are not obvious in the original image (Figs. 6, 7, 9, and 10). The module of the gradient and its angle can in some cases quickly unveil to the eye aspects of the data that cannot be revealed by displaying fluxes. This is illustrated by Figure 7, which shows the same image of a bow shock displayed in three different ways. It is impractical to precisely locate the cusp of the bow shock by using variations of the flux alone (Fig. 7, left). When the module of the gradient of the flux is displayed (Fig. 7, middle), the cusp of the bow shock becomes more easily seen because the module of the gradient goes to zero at the stationary points of flux and this is manifested as a dark "ridge." To distinguish between a maximum, a minimum, and a saddle point, we analyze the Hessian and the Laplacian.

For automated feature extraction one needs to identify the extrema points and to take advantage of the multiscale representation by "tracing" the extrema across different scales. For
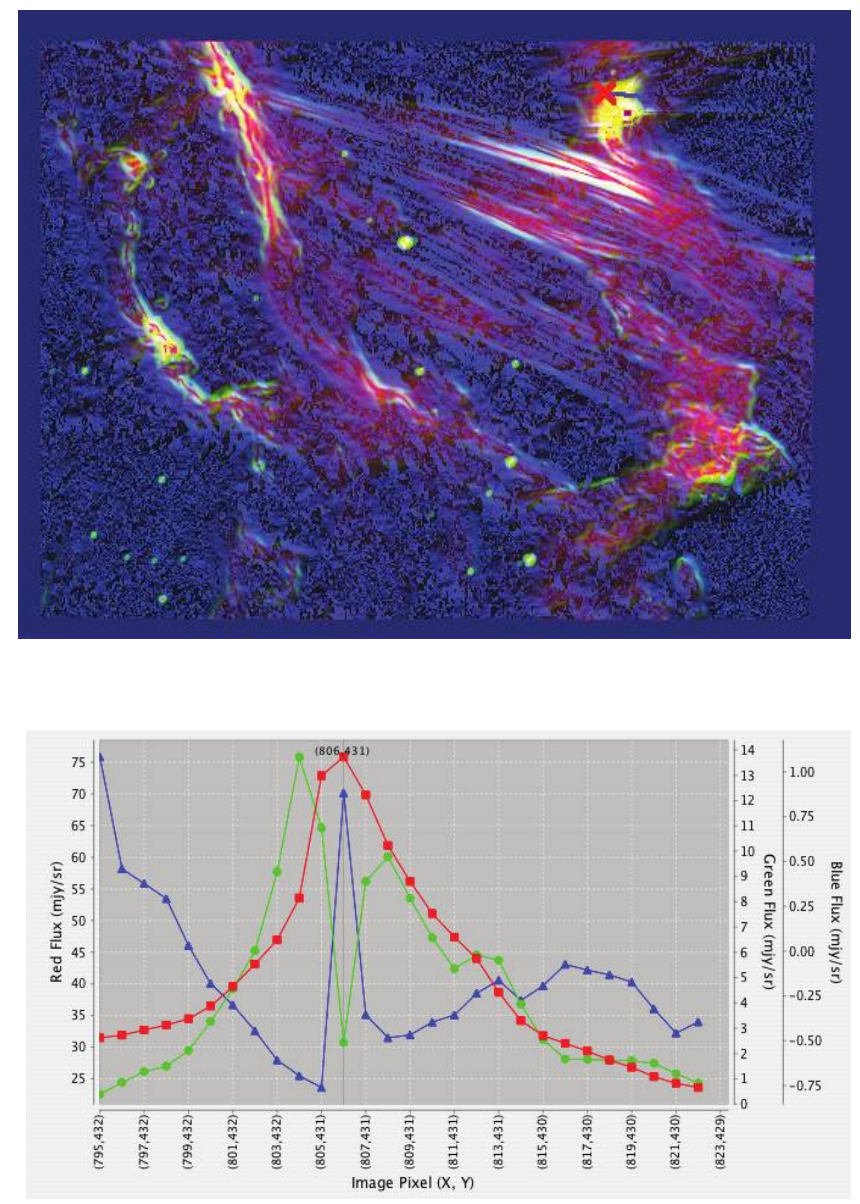

FIG. 8.-Overlaid pre- and postprocessed images. Top: Three overlaid images of IC 405: Spitzer IRAC $8.0 \mu \mathrm{m}$ image, the module of its gradient, and the angle of its gradient (red, green, and blue respectively). The line through the red cross indicates the crosscut. Bottom: Three profiles for the crosscut through " $\times$." The crosshair goes through the ridge point and a local minimum of the module of the gradient. Note the different scales on the left and right vertical axes. 

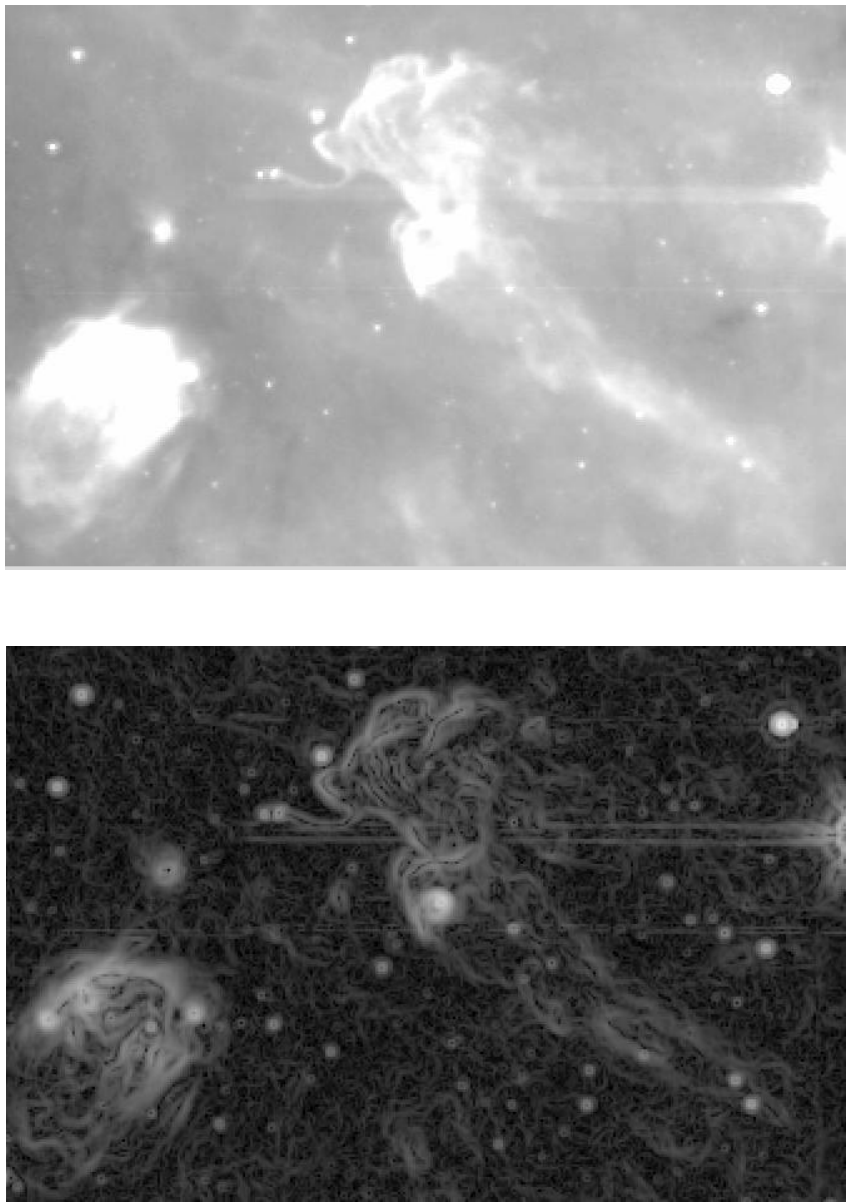

FIG. 9._-Top: Southern Jellyfish Nebula, Spitzer IRAC $8.0 \mu \mathrm{m}$, GLIMPSE, E. Churchwell. The brightness is proportional to the flux (square root scale). Bottom: Morphology of the Southern Jellyfish Nebula image; it reveals fine structure of the diffuse emission (e.g., flux modulations along the ropes) and, at the same time, many localized objects. The brightness is proportional to the module of the gradient of flux (logarithmic scale)

interactive analysis, however, the stationary points can also be useful and the identification of the type of extrema can be done by using a powerful visualization tool provided by our framework. For example, to make sure that the ridges in Figure 7 (middle and right panels) are not spurious, we overlaid the preprocessed and postprocessed (the module of the gradient and the angle of the gradient) images in red, green, and blue in Figure 8 (top) and created crosscuts for the overlaid images (Fig. 8, bottom). The crosshair goes through the ridge point and a local minimum of the module of the gradient. This clearly demonstrates that the ridges have been located precisely.

When applied to images of the Southern Jellyfish Nebula (Mercer et al. 2007; see Fig. 9, top), this approach reveals flux modulations along the ropes (Fig. 9, bottom), which are not obvious in the original image and could indicate waves propagating along the ropes.

These illustrations demonstrate that displaying the module and the angle of the gradient of astronomical images is complementary to displaying the flux and may potentially be helpful in interactive studies of gaseous shocks, jets, and various instabilities. Because this way of looking at astronomical images facilitates morphology studies, "morphology-unveiling operators" (see Figs. 6, 9, 10, and 14) is a more accurate term for astronomical images than "edge detectors," as these operators are
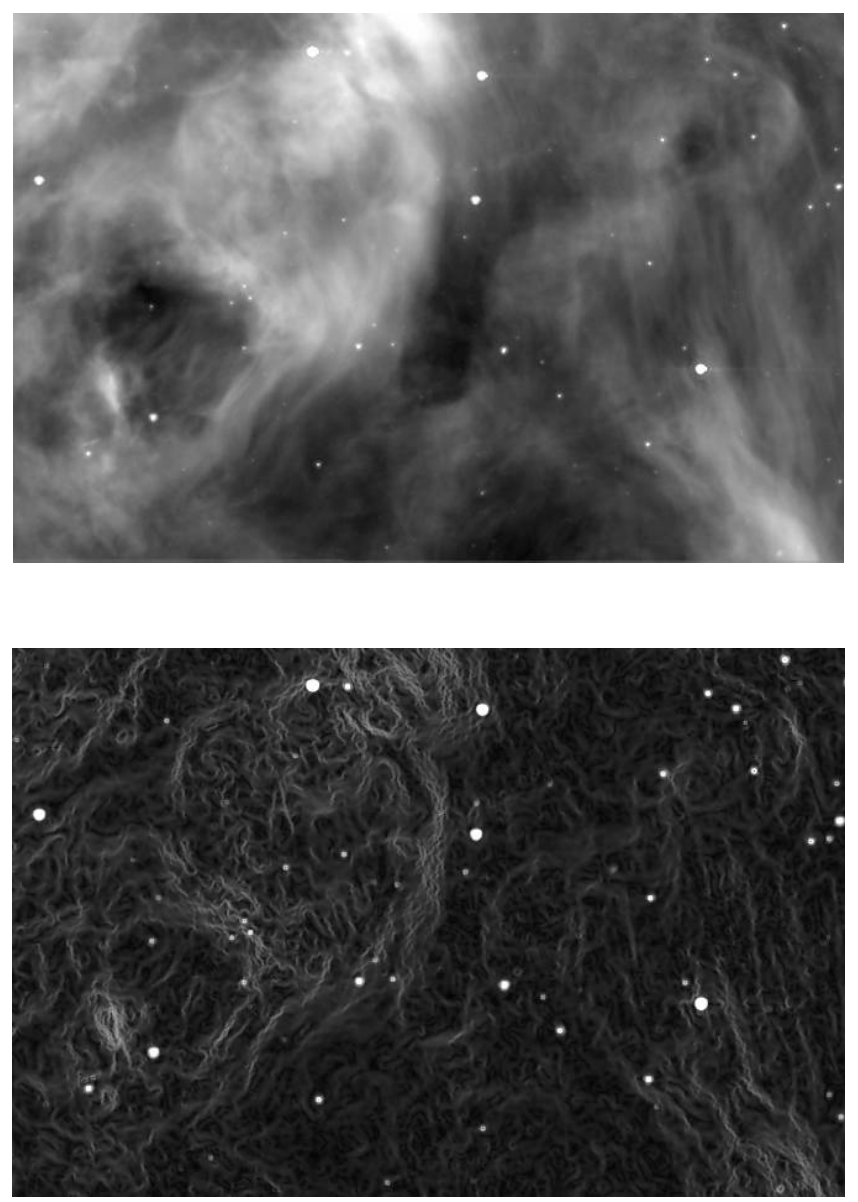

FIG. 10.-Top: HH 34 (center) Spitzer IRAC $8.0 \mu \mathrm{m}$ image, A. NoriegaCrespo. The brightness is proportional to the flux (logarithmic scale). Bottom: HH 34 processed by two operators - the NLD module followed by the morphology unveiling operator; fine structure of the diffuse emission and the same time, many localized objects are brought out. The brightness is proportional to the module of the gradient of flux (square root scale).

called in image processing. Similarly to the way 2D gradients facilitate morphology study and visualization of single-valued images, the multi- and hyperspectral gradients (Sapiro 2001; Tschumperle \& Deriche 2005) will greatly assist visual comprehension of multi- and hyperspectral cubes.

Besides revealing morphology, these operators at the same time better bring many faint point sources out (Figs. 6, 9, and 10). This is because the module of the gradient removes a constant or slowly varying background and also because the module of the gradient of a faint point source may be comparable to the module of the gradient of a bright one, even if their fluxes are drastically different. This capability opens new possibilities for extracting point sources by thresholding in the gradient plane, rather than in the flux plane as it is usually done. We processed images of nebulae with imbedded localized objects (the Crab Nebula, $\eta$ Carina Nebula, and HH 34) and, by using the aforementioned visualization tool, created crosscuts for pre- and postprocessed images. The result for the $\eta$ Carina Nebula is shown in Figure 11. Indeed, as expected, the gradient removes the slowly varying "background."

Since differentiation and convolution are linear operators, LOG is basically a Laplacian of an image that has been convolved with a Gaussian. Our framework facilitates a new way of tackling the problem by creating a module flow that is a sequence of the NLD module and the Laplacian. It is thus becoming a new 

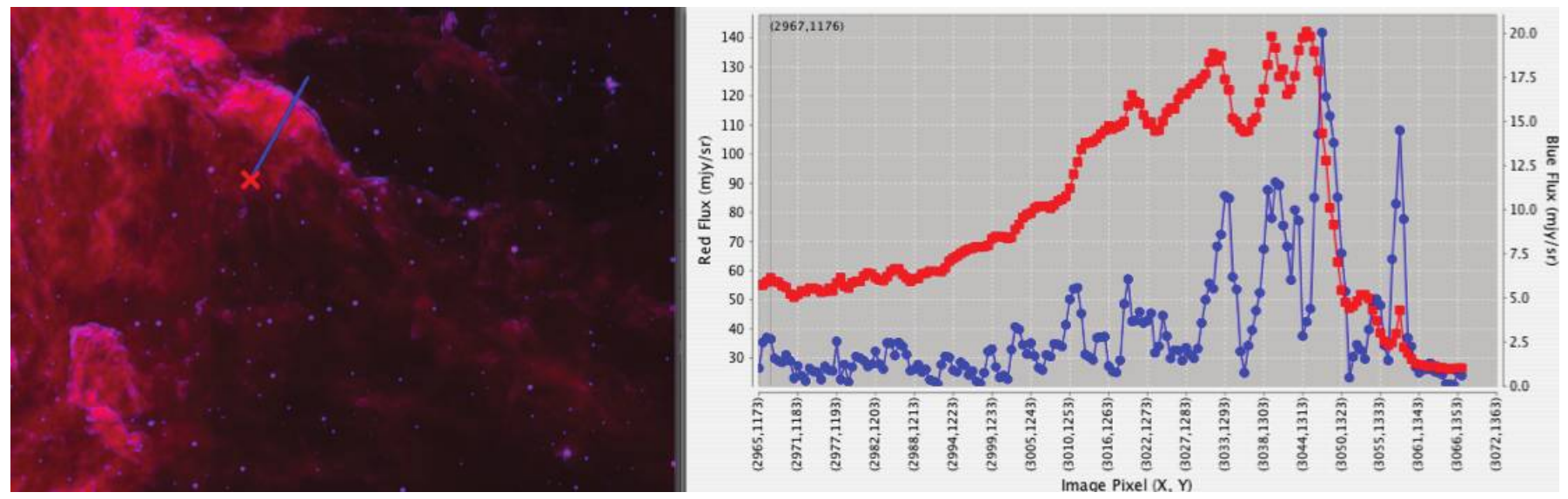

FIG. 11.-Left: $\eta$ Carina Nebula, Spitzer IRAC $8.0 \mu \mathrm{m}$ image, N. Smith. Pre-and postprocessed (by the morphology-unveiling operator) images overlaid in red and blue,

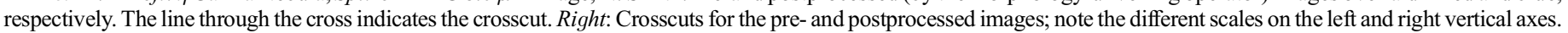

operator-the Laplacian of a nonlinear diffusion (LNLD). As we have demonstrated above, the NLD equation preserves objects better than the convolution with a Gaussian, so LNLD is better suited for locating objects with relatively sharp boundaries. This can potentially be useful, for example, for automated image registration (Hack 2008).

However, if we directly apply the Sobel or Prewitt operators to noisier images such as HH 34 (Fig. 10, top) or G11.2-0.3 (Fig. 3), the main "achievement" is noise amplification (Fig. 12). A straightforward approach to resolving this issue would be to augment smoothing. To do so, we devised an $11 \times 11$ LOG mask (see eq. [8] in the Appendix). Nevertheless, this approach did not lead to a substantial improvement, while preprocessing with the NLD module enabled us to use the morphology-unveiling operator effectively (see Fig. 13, bottom).

Unsharp masking (UM) is a well-known process for sharpening images by subtracting a smoothed version of an image from the original image. We implemented UM by subtracting the median low-pass filtered image form the original one (Fig. 13, top). Comparison of UM with the Sobel operator demonstrates that the former is more sensitive to blobs and sharp edges (Figs. 13 $[$ top $]$ and 14 [bottom] $)$, while the latter is more sensitive to diffuse structures (Figs. 9, 10, and 13, bottom panels).

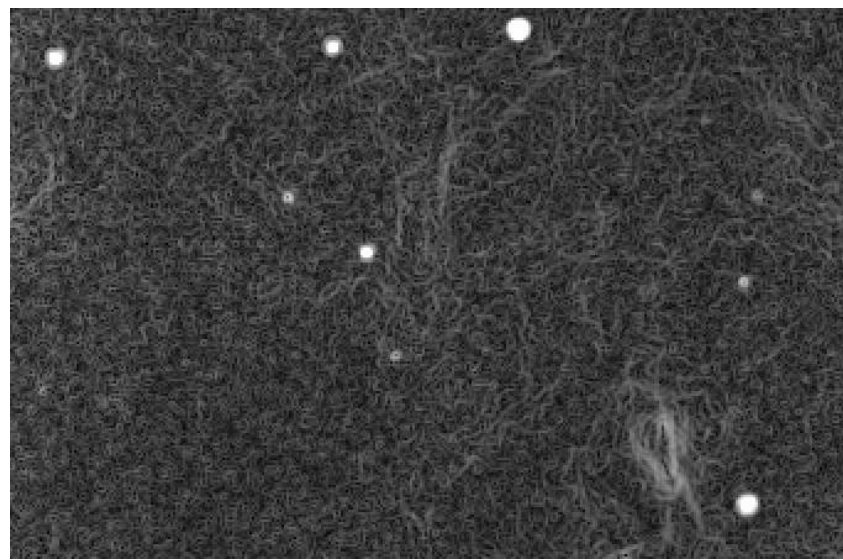

FIG. 12.- HH 34 (center) Spitzer IRAC $8.0 \mu \mathrm{m}$ image, zoomed in; the brightness is proportional to the module of the gradient of flux (logarithmic scale). It is an attempt to look at the fine structure without preprocessing. Cf. Fig. 13.
The introduced operators are complementary to each other and may be used in different combinations with different settings depending on the task. The framework presented here allows one to create interchangeable sequences of operators (called flows), thus facilitating various flexible combinations of different processing operators.
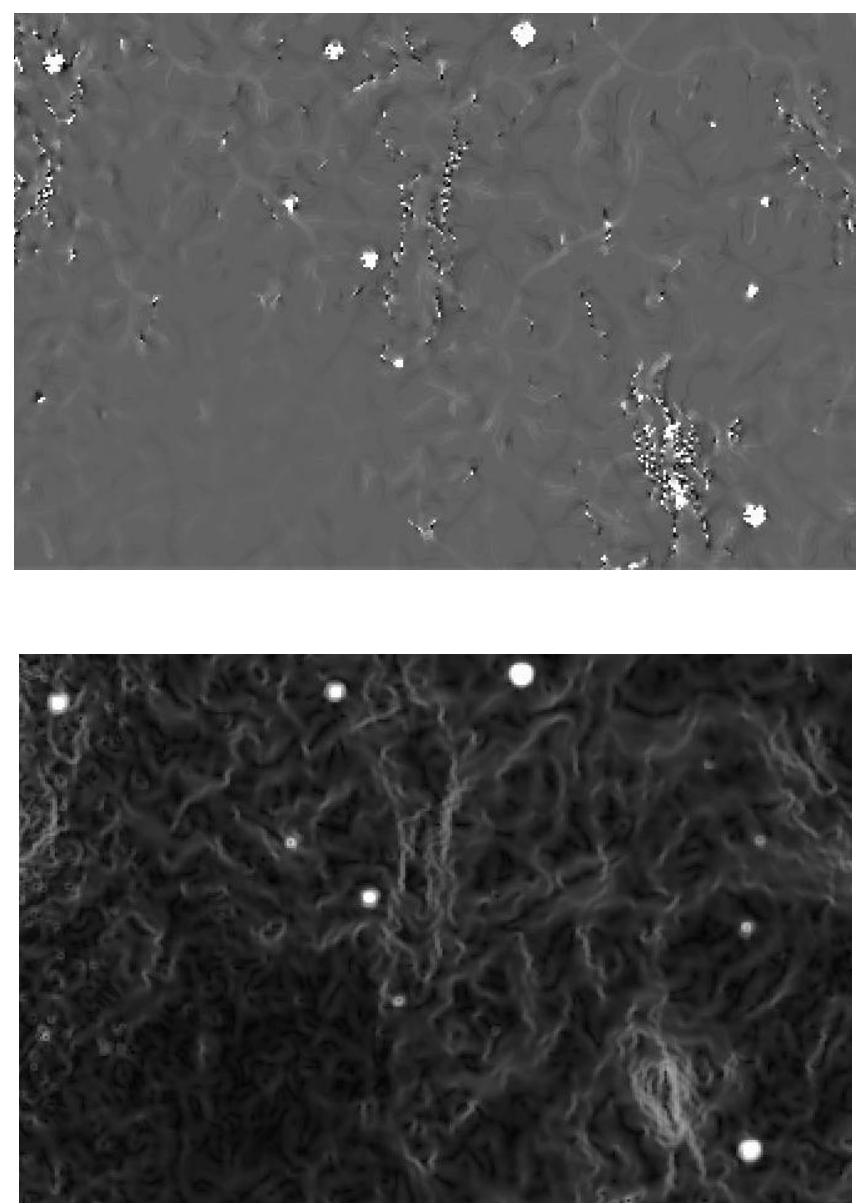

FIG. 13.- Top: HH 34 image, zoomed in; unsharp masking. The brightness is proportional to the flux (logarithmic scale). Bottom: Morphology of HH $34 \mathrm{im}-$ age, zoomed in. The brightness is proportional to the module of the gradient of flux (square root scale). 

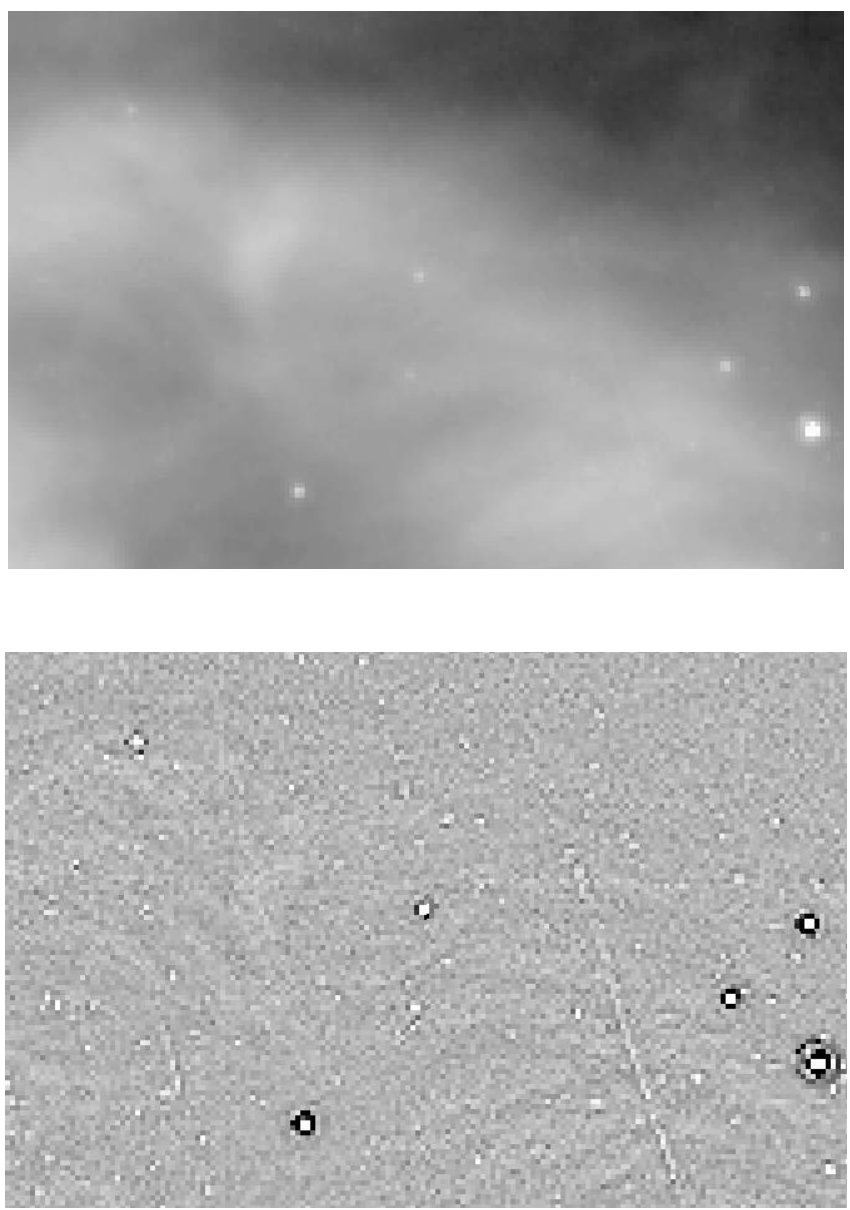

FIG. 14.-Top: HH 34, Spitzer IRAC $8.0 \mu \mathrm{m}$ image; the brightness is proportional to the flux (logarithmic scale). Bottom: HH 34, Spitzer IRAC $8.0 \mu \mathrm{m}$ image; Laplacian of a Gaussian detects radhits that are not obvious in the original image; the brightness is proportional to the flux (logarithmic scale).

The methods described in this and the previous section facilitate interactive analysis and, more importantly, can form a basis for semiautomated detection of different features.

\section{ARTIFACT DETECTION AND SEMIAUTOMATED IMAGE QUALITY ASSESSMENT}

Simply implementing even the most powerful image processing algorithms is not sufficient because quality assessment (QA) should be a part of any image processing framework to monitor and evaluate quality of the processed images and dynamically optimize processing modules.

Moreover, since astronomical data volumes are increasing rapidly, it is clear that manual monitoring of image QA (iQA) for such sets will fail to serve. Indeed, the Large Synoptic Survey Telescope (LSST) alone will be producing about $30 \mathrm{~TB}$ of data per night, so semiautomated iQA is crucial (Tyson 2008). However, existing approaches in astronomy to iQA (not to be confused with the calibration quality assessment) have not even begun to address the complete scope of the problem.

The ultimate goal of image processing is better images, but "better" has no universal quantitative definition and depends on the task, so task-dependent metrics for QA are needed. Existing iQA metrics in the field of image processing are basically variations of a simple mathematical measure called the mean squared
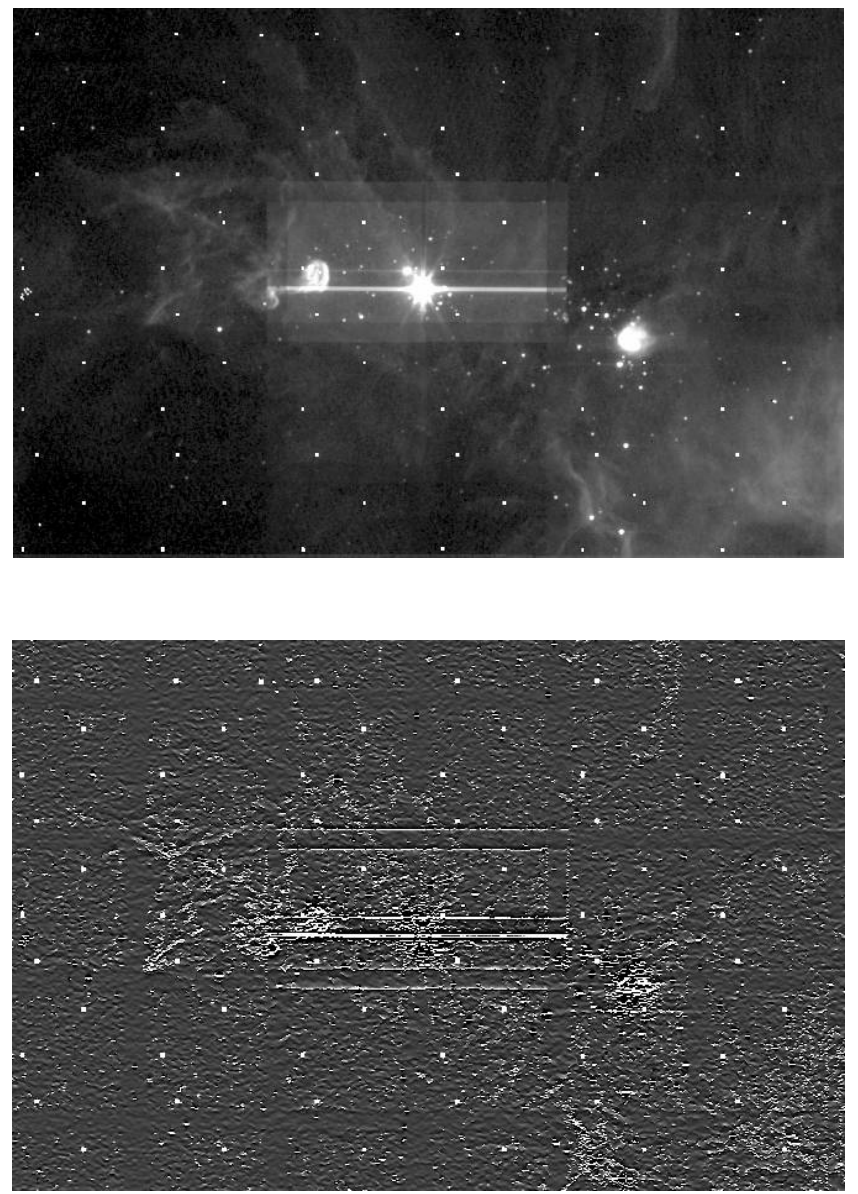

FIG. 15.-Top: Mosaic of NGC 2264, Spitzer IRAC $8.0 \mu \mathrm{m}$; the brightness is proportional to the flux (logarithmic scale). Bottom: Mosaic of NGC 2264, Spitzer IRAC $8.0 \mu \mathrm{m}$, after processing by NLD followed by the shape detector module to detect straight-line artifacts; the brightness is proportional to the flux (logarithmic scale). In addition to bright lines, a faint grid pattern is now discernible (information about such a grid is provided by the Spitzer Science Center together with the mosaic). Such processing can be used for quick iQA screening and flagging "suspicious" images.

error (MSE; Bovik 2005). MSE-based measures, while very sensitive to trivial global image modifications, are insensitive to small details that might be of interest. Moreover, MSE measures make no distinction between noise and blur (which, for example, may be introduced by image processing modules) since they are based on an implicit assumption that image quality is independent of a spatial relationship between image samples.

However, artifacts in astronomical images are often "structured": lines, artificial patterns, patterns of noise, and speckle noise, which is signal-dependent and spatially correlated (see Lafreniere et al. 2007 for important applications). An approach that does not take into account this structural information is not adequate. Our framework implements modules capable of detection of some artificial structures (just a few examples are given in this paper: Figs. 5, 14, and 15), thus forming a basis for semiautomated image quality assessment. That is, the software effectively identifies artifacts and flags images that contain the artifacts.

The task of monitoring image processing is also assisted by the noise estimation module. At this stage of the framework development, this task can be done only interactively, and a way of automating it requires what we call a "smart graphical user 

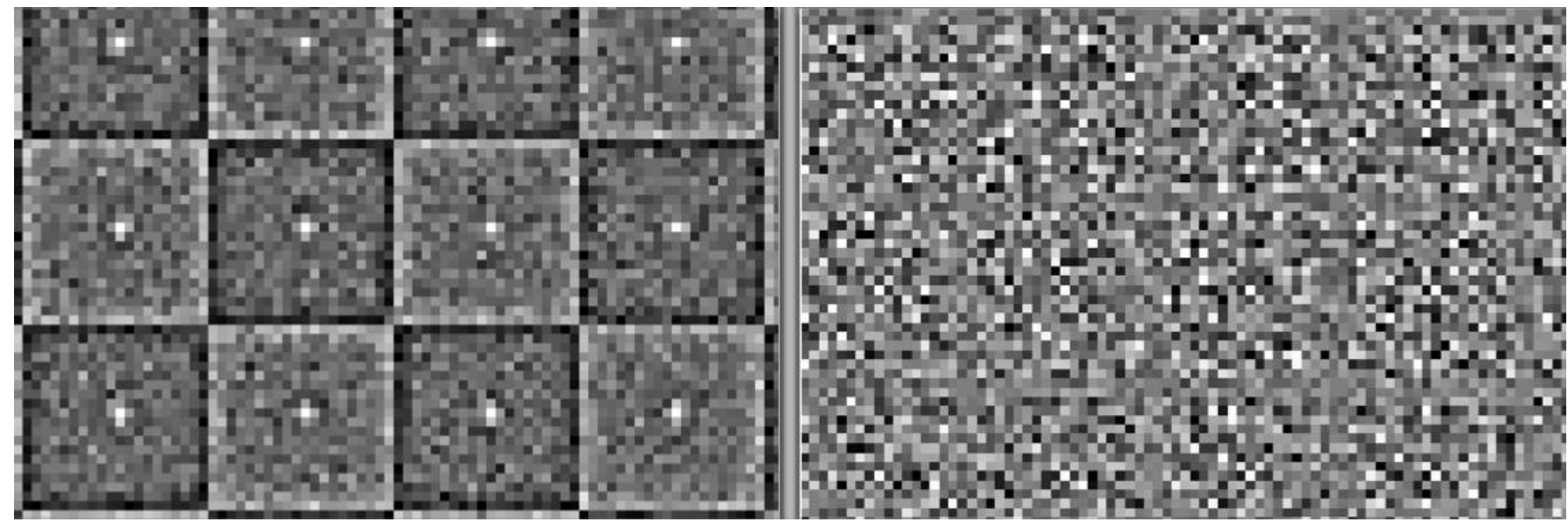

FIG. 16. - Two difference images produced by applying two different noise removal techniques. Left: The difference between the simulated image (Fig. 4, left) and its smoothed version (convolved with a Gaussian, kernel radius $=3$ ); the resulting "noise" retains structures and point sources and is very different from the noise that was added to the chessboard image. Right: The difference between the simulated image (Fig. 4, left) and its smoothed version produced by applying the NLD module (Fig. 4, right). This noise is much more random than the noise in the image on the left.

interface" (GUI) (see $\S 6$ ). As we had discussed earlier, the median and average filters, together with a convolution with a Gaussian, are often used for denoising. By subtracting the filtered image from the original one, the noise and its characteristics are computed. For the multiplicative noise our module for homomorphic filtering ( Lim 1990) is more appropriate. We also compute the standard deviation of the noise from the average of variances measured in a set of image blocks.

Another approach is based on the nonlinear diffusion equation ( $\S 2)$. To compare the NLD approach with the approach based on a convolution with a Gaussian, we used the simulated chessboard image (Fig. 4, left). The noise filtered out by

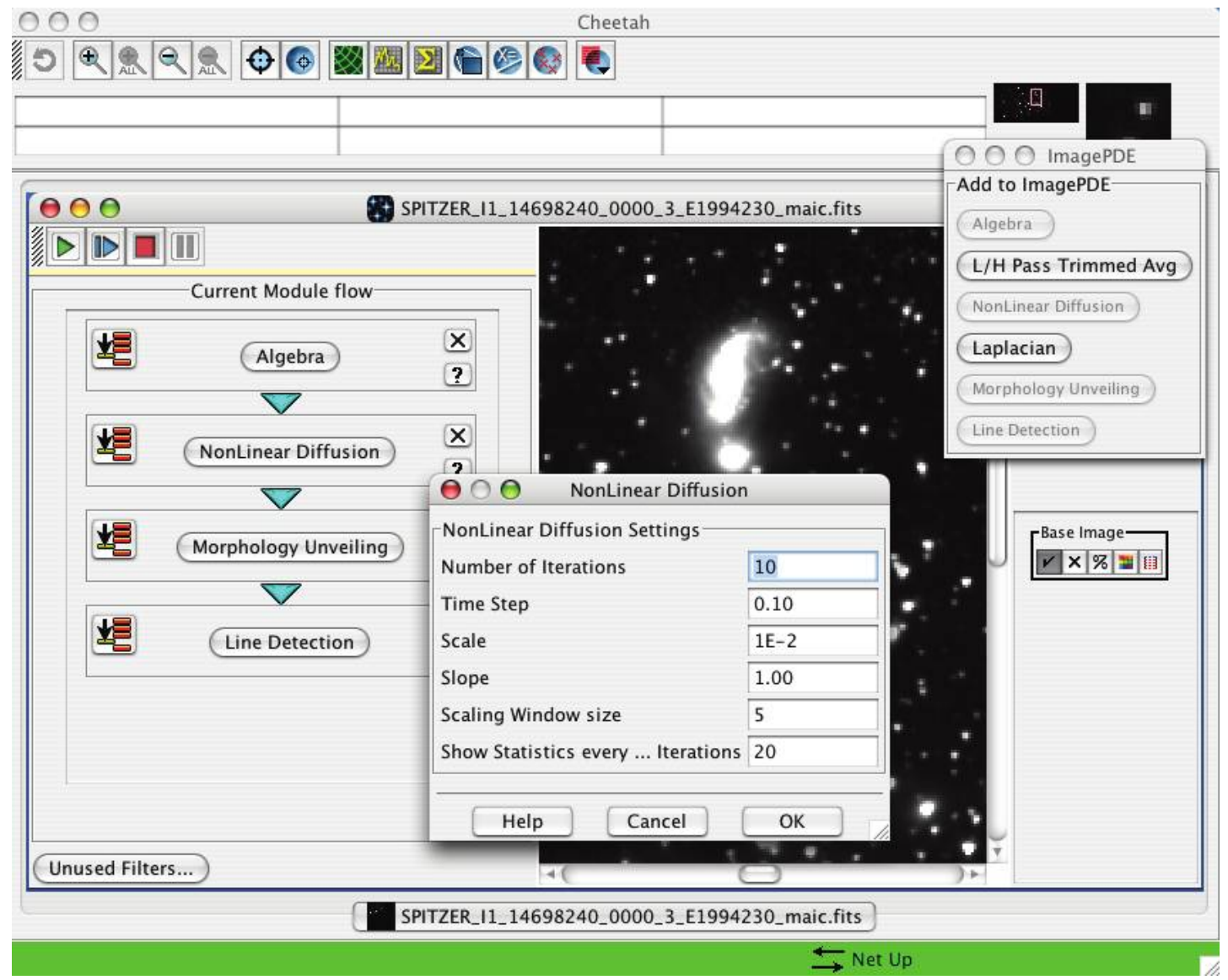

FIG. 17.- Screenshot from the framework. An example of creating a flow: a sequence of four different modules (to the left of the image) to process the image in the window (to the right of the image). In the middle is the settings dialog for the NLD module. 
a convolution with a Gaussian $($ kernel $=3$ ) retained the structures, which have nothing to do with the noise (Fig. 16, left), while the NLD module produced the noise without structures (Fig. 16, right). Thus, the approach based on the NLD is much more efficient at filtering noise, when structures or artifacts are present in the image. Depending on the output of the noise estimation module, the user can rewind the flow, either part way or to the beginning, fine-tune parameters, and run the pipeline again from that point.

Vast data sets demand automated or semiautomated image processing and quality assessment of the processed images. The approach described in this section forms a basis for an interactive, or semiautomated way of iQA. In general, supporting processing and iQA of ever increasing data sets calls for a new approach based on integration of image processing, visualization, and iQA, and the framework presented here is a first implementation of such a synergistic advance.

\section{FRAMEWORK-A BRIEF DESCRIPTION}

Our application framework is built from libraries and reusable components (see $\S 1$ ) developed for the Spitzer Space Telescope's observation planning tool Spot and archive retrieval tool Leopard. ${ }^{1}$ Thus, many components of the framework have already been well tested, so it is very mature, even though it is a new application. The framework provides an integrated environment for processing astronomical images with a very intuitive GUI. It provides many common visualization features plus the ability to combine these images (overlays, etc.) in new and unique ways (see Figs. 8 and 11). The framework allows the user to set up a custom pipeline interactively and to process images singly or in a batch mode (Fig. 17). The final product, as well as the results of each step, are viewable with the framework. The framework provides access to different data archives, is platform-independent, and can easily incorporate custom modules written in any programming language.

\section{CONCLUSION AND FUTURE WORK}

The astronomical community is becoming increasingly aware of the fact that advanced methods of computer vision and image processing should be utilized by astronomers. Indeed, there are more and more groups developing or implementing modern methods of image processing and visualization (Starck et al. 1998, 2002; Martínez \& Saar 2002; Ingalls et al. 2004; Lenzen et al. 2004; Martínez et al. 2005; Martínez 2008; Pesenson et al. 2005a, 2005b, 2007, 2008; Becciani et al. 2007; Borkin et al. 2007; Comparato et al. 2007; Bressert et al. 2007; Goodman et al. 2008).

To bring to the astronomical community these continuing advances, we have implemented some of the modern image processing methods based on nonlinear partial differential equations. We have demonstrated that this approach allows one to reduce noise while preserving point sources and diffuse structures much better than linear standard filtering techniques such as Gaussian convolution, and that it is also superior at noise estimation. We have also shown that second-level operators combined with the PDE-based operators allow one to detect features reliably.

We have also demonstrated that using the module of the flux gradient of an image can efficiently bring out faint point sources,

\footnotetext{
${ }^{1}$ See http://ssc.spitzer.caltech.edu/documents/leopard/ and http://ssc.spitzer .caltech.edu/documents/spot/.
}

thus opening new possibilities for extracting point sources from the module of the gradient plane, rather than from the flux plane as it is usually done. In addition, we have shown that displaying the module and the angle of the flux gradient can reveal important structural features of an astronomical image, which are difficult or impractical to reveal using standard flux visualization. Altogether, these operators facilitate interactive morphology studies and provide complementary means to other techniques such as unsharp masking.

We have also developed a new framework for processing and visualization astronomical images. It is a platform-independent, open architecture application framework that is based on the aforementioned modern developments in the field of image processing. As a framework, it is inherently more flexible and more easily modified to adopt externally written custom software than would be a stand-alone tool. It can operate in both interactive and batch mode.

The framework incorporates a variety of operators, thus enabling a wide parameter space that a user may explore for any particular image (e.g., variable gradient scale parameter $k$, multiple operators that may be run sequentially in different combinations), thus offering the choice of emphasizing features of interest. The example images presented in this paper are not claimed to represent a single best possible result for each image. Indeed, the purpose of the framework is to enable each user to achieve the best results pertaining to that user's particular science goals.

Future developments include the automated recognition and classification of different features; application of the introduced methods to denoising of multi- and hyperspectral images; application of the NLD to speckle noise reducing; implementation of image inpainting algorithms for artifact mitigation and removal of bright sources; utilizing the linear diffusion equation with a spacevarying diffusion coefficient to handle a shift-varying point-spread function; incorporating into the framework frequency domain methods (Fourier analysis, wavelets); developing objective image quality metrics consistent with subjective human evaluation of astronomical images; developing "smart GUI flows" (an ability of a flow to modify itself depending on the intermidiate outputs), thus paving the way to the automated iQA and optimizing processing parameters; and the ability to read very large fits files with small memory footprint.

M. P. would like to thank George Helou, Alberto NoriegaCrespo, William Reach, Isaac Pesenson, Sean Carey, Inseok Song, Joe Chavez, Jim Ingalls, Tom Handley, Michael Egan, Achim Tappe, and Aniello Grado for helpful discussions. The authors would like to thank Loi Ly, Xiuqin Wu, Booth Hartley, and Tatiana Goldina for their contributions to the GUI. M. P. and B. M. thank Seppo Laine for helpful comments on an earlier draft of this paper. The authors would also like to thank the scientific editor for helpful comments and suggestions, and the anonymous referee for helpful suggestions. We would also like to thank the referee for bringing the paper of Chao \& Tsai (2006) to our attention. M. P. would also like to thank Tetsuyasu Uekuma, Dina Pesenson, Igor Pesenson, and Liza Pesenson for discussions and support. This work is based on observations made with the Spitzer Space Telescope, which is operated by the Jet Propulsion Laboratory, California Institute of Technology, under a contract with NASA.

Facility: Spitzer 


\section{APPENDIX}

\section{$11 \times 11$ LAPLACIAN OF GAUSSIAN MASK}

$$
\left(\begin{array}{ccccccccccc}
0 & 0 & 0 & -1 & -1 & -2 & -1 & -1 & 0 & 0 & 0 \\
0 & 0 & -2 & -4 & -8 & -9 & -8 & -4 & -2 & 0 & 0 \\
0 & -2 & -7 & -15 & -22 & -23 & -22 & -15 & -7 & -2 & 0 \\
-1 & -4 & -15 & -24 & -14 & -1 & -14 & -24 & -15 & -4 & -1 \\
-1 & -8 & -22 & -14 & 52 & 103 & 52 & -14 & -22 & -8 & -1 \\
-2 & -9 & -23 & -1 & 103 & 178 & 103 & -1 & -23 & -9 & -2 \\
-1 & -8 & -22 & -14 & 52 & 103 & 52 & -14 & -22 & -8 & -1 \\
-1 & -4 & -15 & -24 & -14 & -1 & -14 & -24 & -15 & -4 & -1 \\
0 & -2 & -7 & -15 & -22 & -23 & -22 & -15 & -7 & -2 & 0 \\
0 & 0 & -2 & -4 & -8 & -9 & -8 & -4 & -2 & 0 & 0 \\
0 & 0 & 0 & -1 & -1 & -2 & -1 & -1 & 0 & 0 & 0
\end{array}\right)
$$

Acton, S. 2005, in Handbook of Image and Video Processing, ed. A. Bovik (Amsterdam: Elsevier)

Becciani, U., Comparato, M., Costa, A., Larsson, B., Gheller, C., Pasian, F., \& Smareglia, R. 2007, in ASP Conf. Ser. 376, Astronomical Data Analysis Software and Systems XVI, ed. R. Shaw, F. Hill, \& D. Bell (San Francisco: ASP), 633

Booch, G., Rumbaugh, J., \& Jacobson, I. 2005, The Unified Modeling Language User Guide (New Jersey: Addison-Wesley)

Borkin, M., Goodman, A., Halle, M., \& Alan, D. 2007, in ASP Conf. Ser. 376, Astronomical Data Analysis Software and Systems XVI, ed. R. Shaw, F. Hill, \& D. Bell (San Francisco: ASP), 621

Bovik, A., ed. 2005, Handbook of Image and Video Processing (Amsterdam: Elsevier)

Bressert, E., Edmonds, P., \& Kowal Arcand, K. 2007, AAS Meeting 210, 76.09

Catte, F., et al. 1992, SIAM J. Numer. Anal., 29, 182

Chao, S.-M., \& Tsai, D.-M. 2006, Pattern Recognition Letters, 27(5), 335

Coles, P., \& Lucchin, F. 1995, Cosmology: The Origin and Evolution of Cosmic Structure (New York: Wiley)

Comparato, M., et al. 2007, PASP, 119, 898

Fayad, M., Johnson, R., \& Schmidt, D. 1999, Building Application Frameworks: Object Oriented Foundations of Framework Design (New York: Wiley)

Forsyth, D., \& Ponce, J. 2003, Computer Vision: A Modern Approach (New York: Prentice Hall)

France, K., McCandliss, S., \& Lupu, R. 2007, ApJ, 655, 920

Gonzalez, R., \& Woods, R. 2002, Digital Image Processing (New Jersey: Prentice Hall)

Goodman, A., et al. 2008, in ASP Conf. Ser. 394, Astronomical Data and Analysis Software and Systems XVII, ed. R. W. Argyle, P. S. Bunclark, \& J. R. Lewis (San Francisco: ASP), in press

Grand, M. Patterns in Java, vol. 1, 1998 (New York: Addison-Wesley)

Hack, W. 2008, in ASP Conf. Ser. 394, Astronomical Data and Analysis Software and Systems XVII, ed. R. W. Argyle, P. S. Bunclark, \& J. R. Lewis (San Francisco: ASP), in press

Ingalls, J., et al. 2004, ApJS, 154, 281

Lafreniere, D., et al. 2007, ApJ, 660, 770

Lenzen, F., Schindler, S., \& Scherzer, O. 2004, A\&A, 416, 391

Lim, J. 1990, Two-Dimensional Signal and Image Processing (New Jersey: Prentice Hall)

Martínez, V. 2008, in ASP Conf. Ser. 394, Astronomical Data and Analysis Software and Systems XVII, ed. R. W. Argyle, P. S. Bunclark, \& J. R. Lewis (San Francisco: ASP), in press
Martínez, V., \& Saar, E. 2002, Statistics of Galaxy Distribution (New York: Chapman \& Hall)

Martínez, V., et al. 2005, ApJ, 634, 744

Mercer, E., et al. 2007, ApJ, 656, 242

Mitra, S., \& Sicuranza, G. 2001, Nonlinear Image Processing (San Diego: Academic Press)

Paragios, N., Chen, Y., \& Faugeras, O. 2006, Handbook of Mathematical Models in Computer Vision (Berlin: Springer)

Perona, P., \& Malik, J. 1987, IEEE Trans. Pattern Analysis and Mach. Intell., 12, 629

Pesenson, M., Makovoz, D., Reach, W., Rho, J., \& Laher, R. 2005a, Proc. SPIE, 5913, 669

Pesenson, M., Moshir, M., Makovoz, D., Frayer, D., \& Henderson, D. 2005b, in ASP Conf. Ser. 347, Astronomical Data Analysis Software and Systems XIV, ed. P. Shopbell, M. Britton, \& R. Ebert (San Francisco: ASP), 153

Pesenson, M., et al. 2007, in ASP Conf. Ser. 376, Astronomical Data Analysis Software and Systems XVI, ed. R. Shaw, F. Hill, \& D. Bell (San Francisco: ASP), 123

Pesenson, M., et al. 2008, in ASP Conf. Ser. 394, Astronomical Data and Analysis Software and Systems XVII, ed. R. W. Argyle, P. S. Bunclark, \& J. R. Lewis (San Francisco: ASP), in press

Rudin, L. 1987, Tech. Rep. 5250 (Pasadena: Caltech)

Rudin, L., Osher, S., \& Fatemi, E. 1992, Physica D, 60, 259

Sapiro, G. 2001, Geometric Partial Differential Equations and Image Analysis (Cambridge: Cambridge Univ. Press)

Starck, J.-L., \& Murtagh, F. 2002, Astronomical Image and Data Analysis (Berlin: Springer)

Starck, J.-L., Murtagh, F., \& Bijaoui, A. 1998, Image Processing and Data Analysis: The Multiscale Approach (Cambridge: Cambridge Univ. Press)

Tschumperle, D., \& Deriche, R. 2005, IEEE Trans. Pattern Analysis and Mach. Intell., 27(4), 1

Tyson, A. 2008, in ASP Conf. Ser. 394, Astronomical Data and Analysis Software and Systems XVII, ed. R. W. Argyle, P. S. Bunclark, \& J. R. Lewis (San Francisco: ASP), in press

Weickert, J. 1998, Anisotropic Diffusion in Image Processing (Stuttgart: Teubner)

Witkin, A. 1983, Proc. Int. Joint Conf. Artif. Intell., 1019 